\title{
ESTIMATE FOR INITIAL TSCHEBYSCHEFF POLYNOMIALS COEFFICIENTS ON A CERTAIN SUBCLASS OF BI-UNIVALENT FUNCTIONS DEFINED BY SĂLĂGEAN DIFFERENTIAL OPERATOR
}

\author{
F. MÜGE SAKAR
}

ABSTRACT. In this present study, we introduce a comprehensive class of analytic and bi-univalent functions defined by Sălăgean operator, and also obtain initial Maclaurin coefficient estimates by using the Tschebyscheff polynomials for this subclass. Furhermore, we solve the Fekete-Szegö problem for functions belonging to this subclass.

2010 Mathematics Subject Classification: 30C45.

Keywords: Tschebyscheff polynomials, Fekete-Szegö problem, analytic and univalent functions, bi-univalent functions.

\section{INTRODUCTION}

Denote by $\mathcal{D}$ the unit disc of the complex plane $\mathcal{D}=\{z: z \in \mathcal{C}:|z|<1\}$, and $\mathcal{A}$ the class of functions analytic in $\mathcal{D}$; satisfying the conditions

$$
f(0)=1 \text { and } f^{\prime}(0)=1 .
$$

Then each function $f$ in $\mathcal{A}$ has the Taylor expansion

$$
f(z)=z+\sum_{n=2}^{\infty} a_{n} z^{n} .
$$

Also let $\mathcal{S}$ be the subclass of $\mathcal{A}$ consisting of functions the form (1) which are univalent in $\mathcal{D}$.

Further, we denote the class of starlike functions in $\mathcal{A}$ by $\mathcal{S}^{\star}$. It is well known that a function $f \in \mathcal{A}$ is in $\mathcal{S}^{\star}$ if and only if

$$
\Re\left(\frac{z f^{\prime}(z)}{f(z)}\right)>0 .
$$


Denote by $\mathcal{K}$ the subclass of $\mathcal{S}$ consisting of convex functions, so that $f \in \mathcal{K}$ if and only if, for $z \in \mathcal{D}$

$$
\Re\left(1+\frac{z f^{\prime \prime}(z)}{f^{\prime}(z)}\right)>0 .
$$

These two classes have been widely and repeatedly studied by various authors. The theory of analytic functions has wide application in many physical problems such as fluid flows, heat conduction, aerodynamics and so on. The concern of this work is the study of particular subclass of analytic and bi-univalent functions defined chosen domain.

If the functions $f$ and $g$ are analytic in $\mathcal{D}$, then $f$ is said to be subordinate to $g$ , written as

$$
f(z) \prec g(z), \quad(z \in \mathcal{D})
$$

if there exists a Schwarz function $w(z)$, analytic in $\mathcal{D}$, with

$$
w(0)=0 \text { and }|w(z)|<1 \quad(z \in \mathcal{D})
$$

such that

$$
f(z)=g(w(z)) \quad(z \in \mathcal{D}) .
$$

If the function $f$ and its inverse $F=f^{-1}$ are univalent in $\mathcal{D}$, we say that the function $f \in \mathcal{A}$ is bi-univalent in $\mathcal{D}$.

Let $\Sigma$ define the class of all bi-univalent functions in $\mathcal{D}$. Someone can see a short history and examples of functions in the class $\Sigma$ in [8]. The Koebe $1 / 4$ Theorem [19] asserts that the image of $\mathcal{D}$ under each univalent function $f \in \mathcal{S}$ contains the disk of radius $1 / 4$. According to this, if $F=f^{-1}$ is the inverse of a function $f \in \mathcal{S}$, then $F$ has a Taylor-Maclaurin series expansion in some disk about the origin. So, from the Koebe 1/4 Theorem (for details, see [8]) each univalent $f$ has an inverse $f^{-1}$ fulfilling

$$
f^{-1}(f(z))=z \quad(z \in \mathbb{D})
$$

and

$$
f\left(f^{-1}(w)\right)=w \quad\left(|w|<r_{0}(f), r_{0}(f) \geq \frac{1}{4}\right) .
$$

On the other hand, $f^{-1}$ is represented by

$$
\begin{aligned}
F(w) & =f^{-1}(w)=w-a_{2} w^{2}+\left(2 a_{2}^{2}-a_{3}\right) w^{3}-\left(5 a_{2}^{3}-5 a_{2} a_{3}+a_{4}\right) w^{4}+\cdots \\
& =w+\sum_{n=2}^{\infty} b_{n} w^{n} .
\end{aligned}
$$

A classical theorem of Fekete-Szegö [9] states that for $f \in S$ of the form (1), the functional $\left|a_{3}-\mu a_{2}^{2}\right|$ satisfies the inequality: 


$$
\left|a_{3}-\mu a_{2}^{2}\right|= \begin{cases}3-4 \mu, & \mu \leq 0 \\ 1+\exp \left(\frac{-2 \mu}{1-\mu}\right), & 0 \leq \mu \leq 1 \\ 4 \mu-3, & \mu \geq 0\end{cases}
$$

Keogh and Merkes [10], in 1969, obtained the sharp upper bound of the FeketeSzegö functional $\left|a_{3}-\mu a_{2}^{2}\right|$ for functions in some subclasses of $\mathcal{S}$. The functional has since received great attention, particularly in many subclasses of the family of univalent functions (see for example, [2], [3], [4], [18], [20]).

In the case of a real variable $t$ on $(-1,1)$, the expressions,

$$
T_{n}(t)=\cos n \theta, \quad t=\cos \theta
$$

uniquely define polynomials of the $n$th degree, so called Tschebyscheff polynomials (see [12]).

The Tschebyscheff polynomials $T_{n}(t), t \in(-1,1)$ have the generating function of the form

$$
\sum_{n=0}^{\infty} T_{n}(t) z^{n}=\frac{1-t z}{1-2 t z+z^{2}} \quad(z \in D) .
$$

Thus, we write

$$
T(z, t)=1+L_{1}(t) z+L_{2}(t) z^{2}+\ldots \quad(z \in D) .
$$

Also it is known that

$$
L_{n+1}(t)+2 t L_{n}(t)+L_{n-1}(t)=0
$$

and

$$
\begin{aligned}
& L_{0}(t)=1 \\
& L_{1}(t)=t, \\
& L_{2}(t)=2 t^{2}-1, \\
& L_{3}(t)=4 t^{3}-3 t,
\end{aligned}
$$

The study of operators plays an important role in mathematics especially in geometric function theory. Recently, the interest in this area has been increasing because it permits detailed investigations of problems with physical applications. 
F. Müge Sakar - Bi-Univalent Functions ...

For example, the differential operator is linked between function theory and mathematical physics. Ruscheweyh in 1975 [16], and then several authors (see [1], [7], [11], [13], [14] and [15]) defined new operators and studied various classes of analytic and univalent functions, which generalise a number of previously well known subclasses and at that times they discovered new subclasses of analytic functions. Sălăgean in 1983 [17] defined the differential operator $D^{k}$ of the class of analytic functions by using the technique of convolution. Many authors have used the Sălăgean operator to define and investigate the properties of certain known and new classes of analytic functions.

In this study we also mentioned a comprehensive class defined by Sălăgean operator. The class introduced in this paper is motivated by the corresponding class investigated in [6]. We establish bounds for the coefficients, and solve the FeketeSzegö problem for the functions that belong to this class. Furthermore, some related corollaries and remarks are also considered.

\section{The SubClass $B_{\Sigma}(\mu, \lambda, t, k)$}

In this part, we present and research the comprehensive subclass $B_{\Sigma}(\mu, \lambda, t, k)$. For $f \in \mathcal{A}$, we also consider the Sălăgean operator [17] which introduced by Sălăgean as follows:

$$
\begin{aligned}
& D^{0} f(z)=f(z), \\
& D^{1} f(z)=z f^{\prime}(z)=D f(z), \\
& D^{k} f(z)=D\left(D^{k-1} f(z)\right), \quad(k \in \mathbb{N}:=\{1,2,3, \ldots\}) .
\end{aligned}
$$

If $f$ is given by (1), then from (3) and (4) we see that

$$
D^{k} f(z)=z+\sum_{n=2}^{\infty} n^{k} a_{n} z^{n}, \quad\left(k \in \mathbb{N}_{0}:=(\mathbb{N} \cup\{0\}),\right.
$$

with $D^{k} f(0)=0$.

Next, we consider the following new subclass of analytic bi-univalent functions defined by Sălăgean operator . 
Definition 1. A function $f \in \Sigma$ is said to be in the class $B_{\Sigma}(\mu, \lambda, t, k), \lambda \geq 1, \mu \geq$ $0, k \in \mathbb{N} \cup\{0\}, z \in D$ and $t \in\left(\frac{1}{2}, 1\right]$ if the following subordination hold

$(1-\lambda)\left(\frac{D^{k} f(z)}{z}\right)^{\mu}+\lambda\left(D^{k} f(z)\right)^{\prime}\left(\frac{D^{k} f(z)}{z}\right)^{\mu-1} \prec T(z, t):=\frac{1-t z}{1-2 t z+z^{2}} \quad(z \in D)$.

and

$(1-\lambda)\left(\frac{D^{k} F(w)}{w}\right)^{\mu}+\lambda\left(D^{k} F(w)\right)^{\prime}\left(\frac{D^{k} F(w)}{w}\right)^{\mu-1} \prec T(w, t):=\frac{1-t w}{1-2 t w+w^{2}} \quad(w \in D)$

where the function $F(w)=f^{-1}(w)$.

Now, we give some special cases as remarks for the case of special parameters.

Remark $1(i)$ For $\mu=1$ and $k=0$, we get the class $\mathcal{B}_{\Sigma}(1, \lambda, t, 0)=\mathcal{B}_{\Sigma}(\lambda, t)$ consists of functions $f \in \Sigma$ satisfying the condition

$$
(1-\lambda) \frac{f(z)}{z}+\lambda f^{\prime}(z) \prec T(z, t)=\frac{1-t z}{1-2 t z+z^{2}}
$$

and

$$
(1-\lambda) \frac{F(w)}{w}+\lambda F^{\prime}(w) \prec T(w, t)=\frac{1-t w}{1-2 t w+w^{2}}
$$

where the function $F=f^{-1}$. This class introduced and studied by Bulut et al. [5].

(ii) For $\lambda=1$ and $k=0$, we get the class $\mathcal{B}_{\Sigma}(\mu, 1, t, 0)=\mathcal{B}_{\Sigma}(\mu, t)$ consists of bi-Bazilevič functions:

$$
f^{\prime}(z)\left(\frac{f(z)}{z}\right)^{\mu-1} \prec T(z, t)=\frac{1-t z}{1-2 t z+z^{2}}
$$

and

$$
F^{\prime}(w)\left(\frac{F(w)}{w}\right)^{\mu-1} \prec T(w, t)=\frac{1-t w}{1-2 t w+w^{2}}
$$

where the function $F=f^{-1}$.

(iii) For $\lambda=1, \mu=1$ and $k=0$; we have the class $\mathcal{B}_{\Sigma}(1,1, t, 0)=\mathcal{B}_{\Sigma}(t)$ consists of functions $f$ satisfying the condition

$$
f^{\prime}(z) \prec T(z, t)=\frac{1-t z}{1-2 t z+z^{2}}
$$

and

$$
F^{\prime}(w) \prec T(w, t)=\frac{1-t w}{1-2 t w+w^{2}}
$$


where the function $F=f^{-1}$.

(iv) For $\lambda=1, \mu=0$ and $k=0$; we have the class $\mathcal{B}_{\Sigma}(0,1, t, 0)=\mathcal{S}_{\Sigma}(t)$ consists of functions $f$ satisfying the condition

$$
\frac{z f^{\prime}(z)}{f(z)} \prec T(z, t)=\frac{1-t z}{1-2 t z+z^{2}}
$$

and

$$
\frac{w F^{\prime}(w)}{F(w)} \prec T(w, t)=\frac{1-t w}{1-2 t w+w^{2}}
$$

where the function $F=f^{-1}$.

Theorem 1. Let the function $f(z)$ given by $(1)$ be in the class $B_{\Sigma}(\mu, \lambda, t, k)$. Then

$$
\left|a_{2}\right| \leq \frac{t \sqrt{2 t}}{2^{k} \sqrt{\left|t^{2}\left[(\mu+2 \lambda)(\mu+1)-4(\mu+\lambda)^{2}\right]+2(\mu+\lambda)^{2}\right|}}
$$

and

$$
\left|a_{3}\right| \leq \frac{t}{3^{k}(\mu+2 \lambda)}+\frac{t^{2}}{3^{k}(\mu+\lambda)^{2}}
$$

Proof. Let $f \in B_{\Sigma}(\mu, \lambda, t, k)$. From (6) and (7), we have

$$
(1-\lambda)\left(\frac{D^{k} f(z)}{z}\right)^{\mu}+\lambda\left(D^{k} f(z)\right)^{\prime}\left(\frac{D^{k} f(z)}{z}\right)^{\mu-1}=1+L_{1}(t) p(z)+L_{2}(t) p^{2}(z)+\ldots
$$

and

$$
(1-\lambda)\left(\frac{D^{k} F(w)}{w}\right)^{\mu}+\lambda\left(D^{k} F(w)\right)^{\prime}\left(\frac{D^{k} F(w)}{w}\right)^{\mu-1}=1+L_{1}(t) q(w)+L_{2}(t) q^{2}(w)+\ldots
$$

for some analytic functions

$$
\begin{gathered}
p(z)=c_{1} z+c_{2} z^{2}+c_{3} z^{3}+\ldots \quad(z \in D) \\
q(w)=d_{1} w+d_{2} w^{2}+d_{3} w^{3}+\ldots \quad(w \in D)
\end{gathered}
$$

where $p(0)=q(0)=0$ and $p(z) \leq 1, q(w) \leq 1$ for all $z \in D$. It is well-known that if $|p(z)|<1$ and $q(w) \mid<1$, then

$$
\left|c_{j}\right| \leq 1 \text { and }\left|d_{j}\right| \leq 1 \quad \text { for all } j \in \mathbb{N} \quad \text { and } \quad z, w \in D
$$

From Eqs. (8) and (9) we have 


$$
(1-\lambda)\left(\frac{D^{k} f(z)}{z}\right)^{\mu}+\lambda\left(D^{k} f(z)\right)^{\prime}\left(\frac{D^{k} f(z)}{z}\right)^{\mu-1}=1+L_{1}(t) c_{1} z+\left[L_{1}(t) c_{2}+L_{2}(t) c_{1}^{2}\right] z^{2}+\ldots
$$

and

$$
(1-\lambda)\left(\frac{D^{k} F(w)}{w}\right)^{\mu}+\lambda\left(D^{k} F(w)\right)^{\prime}\left(\frac{D^{k} F(w)}{w}\right)^{\mu-1}=1+L_{1}(t) d_{1} w+\left[L_{1}(t) d_{2}+L_{2}(t) d_{1}^{2}\right] w^{2}+\ldots
$$

It follows from Eqs. (6) and (7) that

$$
\begin{gathered}
2^{k}(\mu+\lambda) a_{2}=L_{1}(t) c_{1} \\
3^{k}(\mu+2 \lambda)\left[\frac{\mu-1}{2} a_{2}^{2}+a_{3}\right]=L_{1}(t) c_{2}+L_{2}(t) c_{1}^{2} \\
-2^{k}(\mu+\lambda) a_{2}=L_{1}(t) d_{1} \\
3^{k}(\mu+2 \lambda)\left[\frac{\mu+3}{2} a_{2}^{2}-a_{3}\right]=L_{1}(t) d_{2}+L_{2}(t) d_{1}^{2} .
\end{gathered}
$$

From Eqs. (12) and (14), we have

$$
c_{1}=-d_{1}
$$

and

$$
2^{2 k+1}(\mu+\lambda)^{2} a_{2}^{2}=L_{1}^{2}(t)\left(c_{1}^{2}+d_{1}^{2}\right) .
$$

Now by summing (13) and (15) we obtain

$$
3^{k}(\mu+2 \lambda)(\mu+1) a_{2}^{2}=L_{1}(t)\left(c_{2}+d_{2}\right)+L_{2}(t)\left(c_{1}^{2}+d_{1}^{2}\right) .
$$

By using (17) in (18), we obtain

$$
\left[3^{k}(\mu+2 \lambda)(\mu+1)-\frac{2^{2 k+1} L_{2}(t)(\mu+\lambda)^{2}}{L_{1}^{2}(t)}\right] a_{2}^{2}=L_{1}(t)\left(c_{2}+d_{2}\right) .
$$

Also, by considering $L_{2}(t), L_{1}(t)$ and (18) we obtain

$$
a_{2} \leq \frac{\sqrt{2 t} t}{\sqrt{3^{k} t^{2}(\mu+2 \lambda)(\mu+1)-2^{2 k+1}\left(2 t^{2}-1\right)(\mu+\lambda)^{2}}} .
$$




$$
3^{k} 2(\mu+2 \lambda)\left[-a_{2}^{2}+a_{3}\right]=L_{1}(t)\left(c_{2}-d_{2}\right)+L_{2}(t)\left(c_{1}^{2}-d_{1}^{2}\right) .
$$

Then in view of (16) and (17) we obtain the equality (21) from (20)

$$
a_{3}=\frac{L_{1}(t)\left(c_{2}-d_{2}\right)}{3^{k} 2(\mu+2 \lambda)}+\frac{L_{1}^{2}(t)\left(c_{1}^{2}+d_{1}^{2}\right)}{2^{2 k+1}(\mu+\lambda)^{2}} .
$$

Next, in order to find the bound on $\left|a_{3}\right|$, by considering $L_{1}(t)$, we obtain

$$
\left|a_{3}\right| \leq \frac{t}{3^{k}(\mu+2 \lambda)}+\frac{t^{2}}{2^{2 k}(\mu+\lambda)^{2}}
$$

So, we obtain the desired result and the proof is completed.

\section{Fekete-Szegö Inequality for the subclass $B_{\Sigma}(\mu, \lambda, t, k)$}

Our next theorem gives the Fekete-Szegö inequality for functions in the class $B_{\Sigma}(\mu, \lambda, t, k)$.

Theorem 2. Let $f$ given by $(1)$ be in the class $B_{\Sigma}(\mu, \lambda, t, k)$ and $\xi \in \mathbb{R}$. Then

$$
\left|a_{3}-\xi a_{2}^{2}\right| \leq \begin{cases}\frac{t}{3^{k}(\mu+2 \lambda)}, & |\xi-1| \leq\left|\frac{(\mu+1)}{2}-\frac{2^{2 k}\left(2 t^{2}-1\right)(\mu+\lambda)^{2}}{3^{k} t^{2}(\mu+2 \lambda)}\right| \\ \frac{2|1-\xi| t^{3}}{3^{k}\left|t^{2}(\mu+2 \lambda)(\mu+1)-2^{2 k+1}\left(2 t^{2}-1\right)(\mu+\lambda)^{2}\right|}, & |\xi-1| \geq\left|\frac{(\mu+1)}{2}-\frac{2^{2 k}\left(2 t^{2}-1\right)(\mu+\lambda)^{2}}{3^{k} t^{2}(\mu+2 \lambda)}\right| .\end{cases}
$$

Proof. From (19) and (20)

$$
\begin{gathered}
a_{3}-\xi a_{2}^{2}=\frac{L_{1}(t)\left(c_{2}-d_{2}\right)}{3^{k} 2(\mu+2 \lambda)}+\frac{L_{1}^{2}(t)\left(c_{1}^{2}+d_{1}^{2}\right)}{2^{2 k+1}(\mu+\lambda)^{2}}-\frac{\xi L_{1}^{3}(t)\left(c_{2}+d_{2}\right)}{3^{k} L_{1}^{2}(t)(\mu+2 \lambda)(\mu+1)-2^{2 k+1} L_{2}(t)(\mu+\lambda)^{2}} \\
=(1-\xi) \frac{L_{1}^{3}(t)\left(c_{2}+d_{2}\right)}{3^{k} L_{1}^{2}(t)(\mu+2 \lambda)(\mu+1)-2^{2 k+1} L_{2}(t)(\mu+\lambda)^{2}}+\frac{L_{1}(t)\left(c_{2}-d_{2}\right)}{3^{k} 2(\mu+2 \lambda)} \\
=L_{1}(t)\left[\left(h(\xi)+\frac{1}{3^{k} 2(\mu+2 \lambda)}\right) c_{2}+\left(h(\xi)-\frac{1}{3^{k} 2(\mu+2 \lambda)}\right) d_{2}\right]
\end{gathered}
$$

where

$$
h(\xi)=\frac{(1-\xi) L_{1}^{2}(t)}{3^{k} L_{1}^{2}(t)(\mu+2 \lambda)(\mu+1)-2^{2 k+1} L_{2}(t)(\mu+\lambda)^{2}} .
$$

Then, by taking modulus of above equality and using by $L_{1}(t)$ and $L_{2}(t)$, we conclude that

$$
\left|a_{3}-\xi a_{2}^{2}\right| \leq \begin{cases}\frac{t}{3^{k}(\mu+2 \lambda)} ; & 0 \leq|h(\xi)| \leq \frac{1}{3^{k} 2(\mu+2 \lambda)} \\ 2 t|h(\xi)| ; & |h(\xi)| \geq \frac{1}{3^{k} 2(\mu+2 \lambda)}\end{cases}
$$

This completes the proof of Theorem 3.1. 
Taking $\xi=1$ in Theorem 3.1, we get the following consequence.

Corollary 3.2 If $f \in B_{\Sigma}(\mu, \lambda, t, k)$, then

$$
\left|a_{3}-a_{2}^{2}\right| \leq \frac{t}{3^{k}(\mu+2 \lambda)}
$$

Taking $k=0$ in Corollary 3.2 we get the following Corollary 3.3.

Corollary 3.3 If $f \in B_{\Sigma}(\mu, \lambda, t, 0)$, then

$$
\left|a_{3}-a_{2}^{2}\right| \leq \frac{t}{(\mu+2 \lambda)}
$$

\section{REFERENCES}

[1] A. Akgül, New subclasses of analytic and bi-univalent functions involving a new integral operator defined by polylogarithm function, Theory and Applications of Mathematics \& Computer Science 7(2) (2017), 31-40

[2] A. Akgül, The Fekete-Szegö Coefficient inequality for a new class of m-fold symmetric bi-univalent functions satisfying subordination condition, (2017), arXiv preprint arXiv:1711.00742.

[3] M.K. Aouf, R.M. EL-Ashwah, H.M. Zayed, Fekete-Szegö inequalities for pvalent starlike and convex functions of complex order, Journal of the Egyptian Mathematical Society 22 (2014), 190- 196.

[4] S. Bulut, Fekete-Szegö problem for subclasses of analytic functions defined by Komatu integral operator, Arab. J. Math. 2 (2013), 177-183.

[5] S. Bulut, N. Magesh, V.K. Balaji, Initial bounds for analytic and bi-univalent functions, by means of Chebyshev polynomials, J. Class. Anal. in press.

[6] M. Çă̆lar, H. Orhan, N. Yağmur, Coefficient bounds for new subclasses of biunivalent functions, Filomat, 27 (2013), 11651171.

[7] M. Darus, R. Ibrahim, Generalization of differential operator, Journal of Mathematics and Statistics, 4(3) (2008), 138-144.

[8] P.L. Duren, Univalent Functions, in: Grundlehren der Mathematischen Wissenschaften, Band 259, New York, Berlin, Heidelberg and Tokyo, Springer-Verlag, 1983.

[9] M. Fekete, G. Szegö, Eine bemerkung über ungerade schlichte funktionen, Journal of the London Mathematical Society, 2 (1933), 85-89.

[10] F.R. Keogh, E.P. Merkes, A coefficient inequality for certain classes of analytic functions, Proc. Am. Math. Soc. 20 (1969), 8-12. 
F. Müge Sakar - Bi-Univalent Functions ...

[11] Y.C. Kim, H.M. Srivastava, Fractional integral and other linear operators associated with the Gaussian hypergeometric function, Complex Var. Theory Appl. 34 (1997), 293-312.

[12] S. Nobbenhuis, Special functions and polynomials, Institute for Theoretical Physics Utrecht University, Leuvenlaan, 2013.

[13] K.I. Noor, M.A. Noor, On certain classes of analytic functions defined by Noor integral operator, J. Math. Anal. Appl. 281 (2003), 244-252.

[14] K.I. Noor, On new classes of integral operators, J. Nat. Geom. 16 (1999), 71-80.

[15] K.I. Noor, M.A. Noor, On integral operators, J. Math. Anal. Appl. 238 (1999), 341-352.

[16] S. Ruscheweyh, New criteria for univalent functions, Proceeding of the American Mathematicl Society, 49 (1975), 109-115.

[17] G.Ş. Sălăgean, Subclasses of univalent functions, Complex Analysis-Fifth Romanian-Finnish seminar, Part 1 (Bucharest, 1981), Lecture Notes in Math., Springer, Berlin, vol. 1013 (1983), 362-372.

[18] H.M. Srivastava, A.K. Mishra, M.K. Das, The Fekete-Szegö problem for a subclass of close-toconvex functions, Complex Variables Theory Appl. 44 (2001), 145-163.

[19] H.M. Srivastava, A.K. Mishra, P. Gochhayat, Certain subclasses of analytic and bi-univalent functions, Appl. Math. Lett. 23 (2010), 1188-1192.

[20] P. Zaprawa, On Fekete-Szegö problem for classes of bi-univalent functions, Bull. Belg. Math. Soc. Simon Stevin, 21 (2014), 169-178.

F. Müge Sakar

Department of Business Administration,

Faculty of Economics and Administrative Sciences,

Batman University,

Batman, Turkey

email: mugesakar@hotmail.com 